\title{
Meta-analysis using a Lehmann classifier of obligate seeding species germination in fire-prone Mediterranean Type environments
}

\section{Andreas $Y$ Troumbis ( $\square$ atro@aegean.gr)}

University of the Aegean School of the Environment: Panepistemio Aigaiou Schole Periballontos https://orcid.org/0000-0003-3884-9141

\section{Research Article}

Keywords: Meta-analysis, Classification, Seed germination, Pyrophytism, Fire ecology, Cistaceae

Posted Date: August 10th, 2021

DOI: https://doi.org/10.21203/rs.3.rs-554170/v1

License: (c) (i) This work is licensed under a Creative Commons Attribution 4.0 International License.

Read Full License 
$4 \quad$ Meta-analysis using a Lehmann classifier of obligate seeding species

5 germination in fire-prone Mediterranean Type environments

a: contact person: University Hill, Mytilene, Lesbos Island, 81100, Greece; cell phone: +30

\section{Declarations:}

15 No conflict of interest to report

16 No funding received for this research

17 AYT is the only contributor to this research (from conception to submission)

18 No ethical issues (experimentation on Humans and/or Animals)

19 
The generalization that specific seed traits such as dormancy, longevity, or heat-triggered germination of plant species expanding in pyrogenic environments where stochastically but recurrently fire disturbance occurs is a fitness increasing adaptation of obligate seeders dates from the early $20^{\text {th }}$ century. During the last few decades, this hypothesis, qualified as a pyrophytic strategy, is re-evaluated under the lenses of conservation biology and climate change research. The validity of pyrophytism as an equilibrium response to fire vs. the interpretation that the obligate seeding strategy is instead an opportunistic or generalist response to the multitude of abiotic and biotic factors determining the variability and heterogeneity of fire-prone environments such as the Mediterranean Type Ecosystems is indirectly examined and narratively promoted in the renewed fire ecology literature. In this paper, I suggest a need for a typified meta-analysis of the abundant but disparate wealth of research protocols and data to achieve a quantitatively strict understanding of the limits of the contrasting hypotheses. I develop a meta-analytic classifier and test its feasibility and applicability across taxonomic, biologic, and ecological levels of organization, i.e., from the intra-population or inter-individual local level progressively to inter-genus and intra-family levels, across the Mediterranean Basin. Cistaceae species, emblems of the Mediterranean shrublands, are the model for this research. The results of this exercise support the feasibility and flexibility of the Lehmann-type classifier developed. Although Cistus species do respond positively to heat-shocks at the local level, significant variability is uncovered among higher taxa levels and furthermost as the environmental variability increases. The germination variability complicates generalizations when climatic variability and change come into play, questioning long-standing 'certitudes' and Mediterranean forest managers and conservation planners' practices.

\section{Keywords}

47 Meta-analysis; Classification; Seed germination; Pyrophytism; Fire ecology; Cistaceae 


\section{Introduction}

The idea of plant species adapting to stochastic but recurring fires in fire-prone environments dates from the early $20^{\text {th }}$ century. In the Continental European phytosociological tradition, Kuhnholtz-Lordat (1938) has even coined the term 'pyrophytism' (e.g., Trabaud 1994; Troia and Laguna 2015) to qualify -and classify- species into broad categories of recovery strategy from a fire. These are (1) the hard-coated obligate seeders such as members of the Cistaceae family that recover through heat-activated dormant seeds, and (2) the resprouters such as Quercus coccifera that recover mainly through activated dormant vegetative buds (e.g., Luna et al. 2007). After decades of relative marginalization as an ecological research priority, the 'pyrophytism' issue regains interest within broader climate change research (e.g., Ooi 2012; Hudson et al. 2015; Chamorro et al. 2017). During the intermediate period, disparate efforts focused on optimizing technical protocols on studying supposedly fire-dependent biological traits of species and their seeds' physiology (refer to Luna (2020) for a comprehensive report on these issues). The incumbent prediction is that fire outbursts' frequency will increase as extreme disturbance events multiply (e.g., Marlon 2020).

One main issue from the perspective of fire ecology is the distinction between the interpretation of fire-dependent -and other fire cues such as smoke and water stress (e.g., Chamorro and Moreno 2019) - seed germination and subsequent recruitment of seedlings as an equilibrium response to fire vs. the interpretation of post-fire fitness increase as a consequence of equilibrium responses of species to abiotic and biotic factors in fire-prone ecosystems (high-energy flows, landscape heterogeneity, granivory and herbivory (e.g., Zavala et al. 2000), aridity (e.g., Luna and Chamorro 2016; Chamorro et al. 2017), soil characteristics and 3D variability of temperature shocks above- and below- ground (e.g., Luna et al. 2019). In the case of obligate seeding species, the post-fire dynamics that co-determine vegetation recovery and change are essential concerning climate change effects (e.g., Fyllas and Troumbis 2009; Zogas et al. 2020) and the diversity-productivity relationship (e.g., Troumbis and Memtsas 2000). Within this epistemic framework, a methodology able to differentiate the two interpretations is both important as an evolutionary question per se regarding flammability (e.g., Troumbis and Trabaud 1989; Gagnon et al. 2010) or seed germination niche (e.g., Luna et al. 2007), and as a practical issue for species conservation and ecosystem restoration challenges (e.g., Fyllas et al. 2008; Driscoll et al. 2010).

The wealth of relevant literature amassed (for a synthetic description, see Figure 1) poses the problem of meta-analysis and synthesis of data accumulated across taxa, biomes, changing environmental conditions, and combinations of disturbances (e.g., Camac et al. 2015). Various approaches have been proposed to that end for specific species-populationenvironment continua (narrative, e.g., D’Antonio and Flory 2017; statistical modeling, e.g., Dijkstra and Adams 2015). There is a need for a method encompassing heterogeneity of protocols and data, standardizing random error incorporation in meta-analysis attempts.

In this paper, I attempt to develop such a method based on a classification procedure for continuous or ordered categorical germination diagnostic tests values for which the test is positive. I make use of the heritage of meta-analysis literature from machine learning, atmospheric sciences, medicine, psychology, or finances on the Receiver Operating Characteristic (ROC) classifier analysis (e.g., Fawcett 2006), the Summary Receiver Operating Characteristic (SROC) curves (e.g., Holling et al. 2012) and the foundational textbook on applications of Bayesian statistics in Ecology by Hilborn and Mangel (1997).

The rationale of the paper develops in two stages. First, the mean structure model for a single experiment presents the relationship between seed germination conditional probabilities given their viability. Second, extensions and variations of the mean structure model for multiple 
compared similar experiments are analyzed. I test this theory through indicative experimental results that sequentially develop in a taxonomic scale.

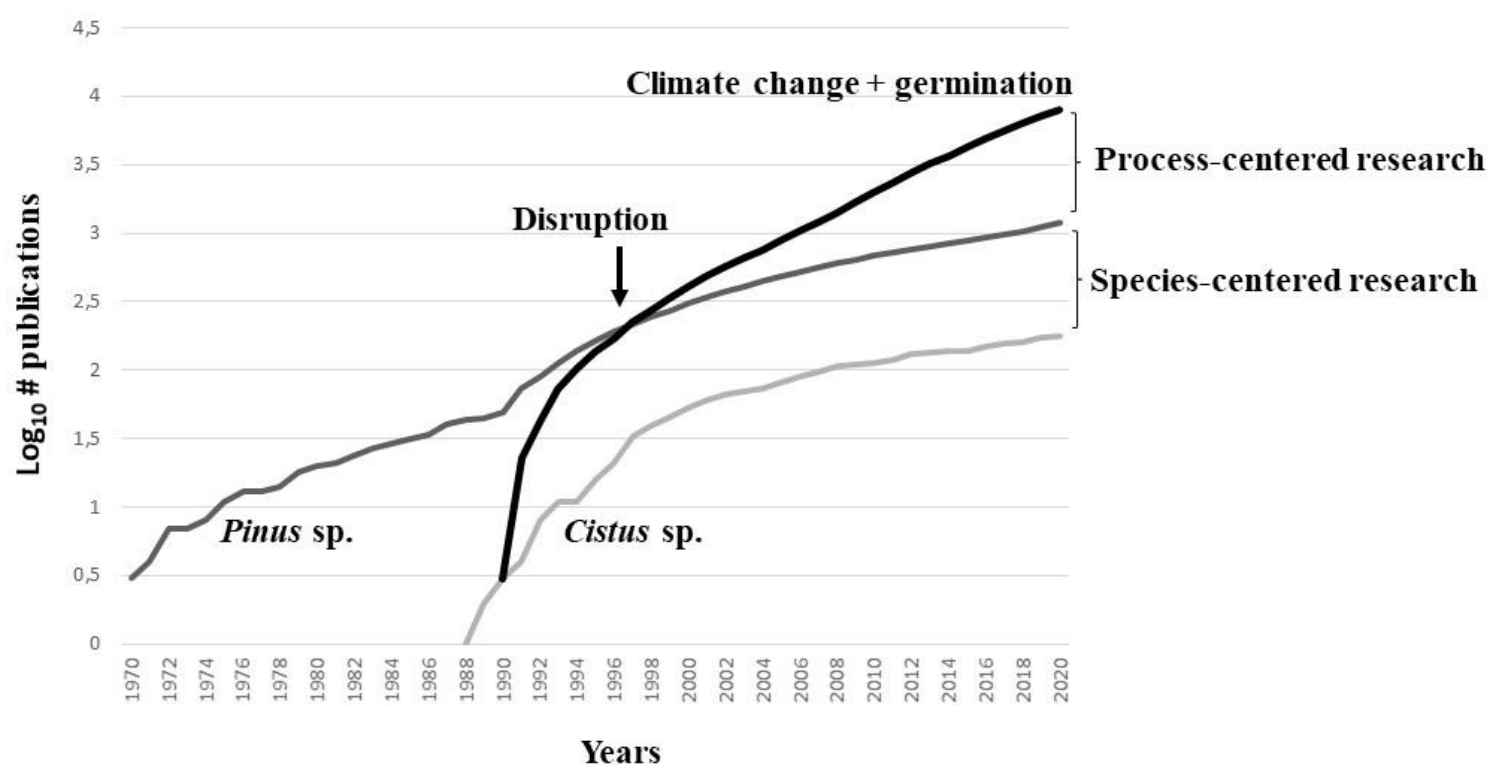

Figure 1: Evolution of research effort and orientation on seed germination of pyrophytic emblems, measured as the number of publications recorded in the Web of Knowledge/Science ( $\log _{10}$ scale). Climate change-related research on germination appears in 1990, coinciding with the publication of the first IPCC (Intergovernmental Panel on Climate Change) report. Research disruption occurred in the middle of the '90s.

\section{A theoretical classifier based on a Lehmann model}

\subsection{Single experiment}

Let us consider $i=1 \ldots k$ samples of $n_{i}$ seeds/sample of some obligate seeder species submitted to germination tests (see the section on the experimental protocol for treatments and species). At the end of the germination test, non-germinated seeds are checked for viability. Four categories of seeds/sample are then defined, and results obtained/sample are identified quantitatively: (1) germinated seeds, $g_{i}=\frac{x_{i}}{n_{i}} ;$ (2) non-germinated seeds $f_{i}=\frac{\left(n_{i}-x_{i}\right)}{n_{i}}=\frac{y_{i}}{n_{i}}$; (3) non-viable seeds, $d_{i}=\frac{m_{i}}{y_{i}}$; and (4) viable but non-activated seeds, $v_{i}=\frac{y_{i}-m_{i}}{y_{i}}$. Considering that seed viability is a precondition for germination, $g_{i}$ corresponds to conditional probabilities of $P(+\mid+)=\hat{g}_{i}=\frac{x_{i}}{n_{i}}$ measuring the sensitivity of a species by treatment combination or 'true positive germination rate'. If 'non-germination' is called specificity in a species by treatment combination, then 'false germination rate' corresponds to $P(-\mid+)=\left(1-\hat{v}_{i}\right)=\left(1-\frac{\hat{y}_{i}-\hat{m}_{i}}{n_{i} \hat{f}_{i}}\right)$. The probability $P(-\mid-)=\frac{\hat{m}_{i}}{y_{i}}$ corresponds to the 'true negative germination rate'. 
Since $\hat{g}_{i} \hat{f}_{i}$ and $\hat{v}_{i}$ are continuous variables, pairs of values $\left(\frac{x_{i}}{n_{i}}, 1-\frac{y_{i}-m_{i}}{n_{i} f_{i}}\right)$ serve to

125 Therefore, $\theta=\frac{\log g}{\log v}=\frac{\log \left(\frac{x_{i}}{n_{i}}\right)}{\log \left(\frac{y_{i}-m_{i}}{n_{i} f_{i}}\right)}$. Further, Hastie et al. (2009) $c$-statistic, i.e. construct sensitivity-specificity curves. In terms of Lehmann models' family (1990, p. 163) and Le (2006) demonstration of the relationship between sensitivity and specificity, the equation $g=v^{\theta}$ is applicable if $g \in[0,1], v \in[0,1], \theta>0$. These conditions are satisfied $d e$ facto in the seed germination test case.

$c=\int_{0}^{1} v^{\theta} d v=\frac{1}{1+\theta}$, calculates the surface under the potential sensitivity-specificity curve as a metric of the seed bank recovery potential.

This procedure fits the needs of a single adequately designed experiment that could lead to generalization, overcoming the usual narrative approach of result interpretation and discussion. Figure 2 presents the theoretical sensitivity-specificity curves for different values of the metric $\theta$.

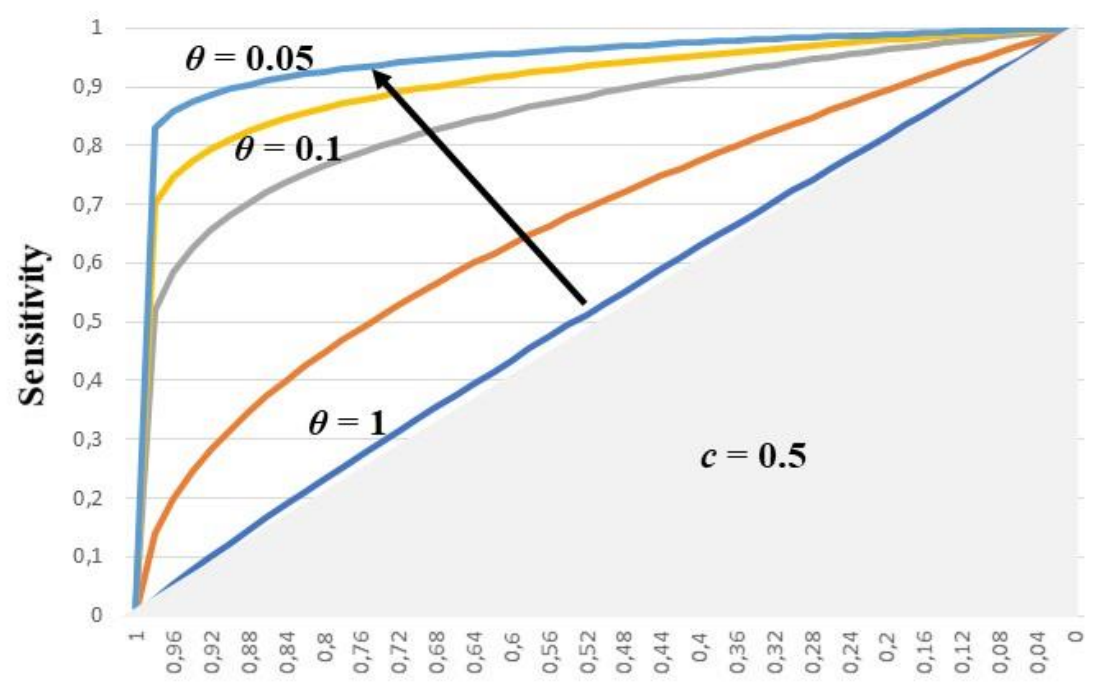

\section{1-Specificity}

Figure 2: Theoretical sensitivity - (1-specificity) curves for different values of the metric $\theta$. For $\theta=1$, the diagonal line $[(0,1),(1,0)]$ indicates a value of $c$-metric of the surface under the diagonal line $=0.5$; it corresponds to the grey surface or area under the curve in the sensitivity vs. 1 -specificity plane. Notice that the smaller $\theta$ is, the larger the value of $c$. The 1 -specificity scale in the $\mathrm{x}$-axis is adopted for compatibility with the classic ROC curve presentation. 


\subsection{Variations of the Lehmann-type classifier}

What are the characteristics of the sequence of trials classifier $\theta$ developed in the previous section? First, its invariant character, i.e., one pair $\left(\hat{g}_{i}, \hat{v}_{i}\right)$ per experiment $i$, or in other words, one pair of true positive vs. false positive germination rates observed. However, different pairs could have different specificity and sensitivity values and still reflect the same diagnostic accuracy. The second characteristic is typical within a log-space domain of application.

One should examine potential extensions and variations of the basic $g=v^{\theta}$ model to adapt it to $k$ compared experiments of similar protocols and/or applications within logit-space. The latter confers an interesting property since within the log-space, $\theta>0$, whereas within the logit space, $\theta \in[0,1]$. Knowing from conditional probability theory that the probability

$P(+\mid+)=\hat{g}$ and $P(-\mid+)=\hat{v}$, the basic model $g=v^{\theta}$, where $\theta=\frac{\log g}{\log v}=\frac{\log \left(\frac{x_{i}}{n_{i}}\right)}{\log \left(\frac{y_{i}-m_{i}}{n_{i} f_{i}}\right)}$, the logit of the probability $\hat{g}$ is transformed as

$\operatorname{logit}(g) \quad=\log \frac{g}{1-g}=\log g-\log (1-g)=-\log \left(\frac{1}{g}-1\right)$, i.e., the logartithm of the corresponding odds. For the two probabilities, the difference of their logits is the logarithm of their ratio $\alpha$ :

$\log \alpha=\log \left(\frac{g / 1-g}{v / 1-v}\right)=\log \left(\frac{g}{1-g}\right)-\log \left(\frac{v}{1-v}\right)=\operatorname{logit}(g)$-logit $(v)$. Therefore, in the logspace, $\alpha$ expresses the ratio of true positive by false negative germination rates, whereas, in the logit-space, it expresses the difference between the true positive-false negative germination rates (Table 1).

Table 1: Recapitulation of various extensions of the sequence of trials classifier $\theta$ and their relations with the basic Lehmann model $g=v^{\theta}$.

\begin{tabular}{|c|c|c|c|c|c|c|c|}
\hline \multicolumn{4}{|c|}{ Model formulation } & Space & Variables & Classifier(s) & Parameters \\
\hline \multicolumn{4}{|c|}{$\log g=\theta \log v$} & $\log$ & $g, v$ & $\theta$ & \\
\hline \multicolumn{4}{|c|}{$\log g=\alpha+\theta \log v$} & $\log$ & $g, v$ & $\theta$ & $\begin{array}{l}\alpha: \text { ratio true positive/false } \\
\text { negative germination rates }\end{array}$ \\
\hline $\log$ & $\frac{g}{1-g}$ & \multicolumn{2}{|l|}{$=\theta \log [\overline{1-v}$} & Logit & $g, v$ & $\theta$ & \\
\hline $\log$ & $\frac{g}{1-g}$ & $=\alpha+\theta \log [$ & $\left.\frac{v}{1-v}\right]$ & Logit & $g, v$ & $\theta$ & $\begin{array}{c}\alpha: \text { difference true } \\
\text { positive-false negative } \\
\text { germination rates }\end{array}$ \\
\hline \multicolumn{4}{|c|}{$\log g_{i}=\left(\theta_{i}+\alpha_{i}\right) e^{\beta}$} & Logit & $g, v$ & $\theta$ & $\alpha, \beta$ \\
\hline
\end{tabular}


This situation implies between-experiment heterogeneity of specificity and sensitivity results, and therefore, it is necessary to consider the variances of $\hat{g}, \hat{v}$ and $\hat{\theta}$.

Let $z_{i}=\log x_{i}-\log n_{i}$ is the $\log$-true germination rate and $w_{i}=\log \left(y_{i}-m_{i}\right)-\log n_{i} f_{i}$, the log-false positive germination rate, given that $x_{i}>0, y_{i}>0, m_{i}>0$, for $i=1 . . k$ similar experiments. Further, the estimated variances for $\log \left(g_{i}\right)$ and $\log \left(v_{i}\right)$ are given by:

$E\left(\operatorname{VAR}\left(\log \hat{g}_{i}\right)\right)=E\left(\operatorname{VAR}\left(\log \left(x_{i} / n_{i}\right)\right)=s_{i}^{2}=\frac{1}{x_{i}}-\frac{1}{n_{i}}\right.$

$E\left(\operatorname{VAR}\left(\log \hat{v}_{i}\right)\right)=E\left(\operatorname{VAR}\left(\log \left(y_{i}-m_{i} / n_{i} f_{i}\right)\right)=t_{i}^{2}=\frac{1}{y_{i}-m_{i}}-\frac{1}{n_{i} f_{i}}\right.$

Assuming that in seed germination protocols, sample size/experiment(s) are moderately large, at least, it is acceptable to adopt a bivariate normal distribution for $\log \hat{g}$ with mean: $\log g$ and variance: $\sigma_{g}^{2}$, and equivalently $\log v$ and $\sigma_{v}^{2}$ for $\log \hat{v}$. The covariance would be $\sigma$ and Pearson's correlation coefficient $\rho=\frac{\sigma}{\left(\sigma_{g} \sigma_{v}\right)}$. Under typical conditionality conditions $\log g_{\mid \log v}$, it applies that $E(\log \hat{g} \mid \log \hat{v})=\log g+\rho \frac{\sigma_{g}}{\sigma_{v}}(\log (\hat{v})-\log (v))$ which can be rewritten in the form $\alpha+\theta \log (\hat{v})$, where $\alpha=\log (g)-\theta \log (v)$ the test's diagnostic accuracy and $\theta=\rho \frac{\sigma_{g}}{\sigma_{v}}$. For values of $\alpha=0$, the original equation arises in a log-space.

What if another, more inclusive, model might be preferable, such as $\log g_{i}=\log \left(v_{i}+\alpha_{i}\right) e^{\beta}$, following, e.g., Rutter and Gatsonis (2001)? Using the notation adopted here above, the alternative model becomes $\log g_{i}=\left(\theta_{i}+\alpha_{i}\right) e^{\beta}$ and $\log v_{i}=\theta$ where $\alpha$ expresses the test's diagnostic accuracy as the difference between log-true positive and log-false negative germination rates. The basic $g=v^{\theta}$ model is just a particular case, when $\beta=0$ and $\alpha$ is the ratio between them.

\section{Empirical comparisons based on the Lehmann-type classifier}

In the following sections, I use relevant examples of both published research and personal unpublished yet data to assess the Lehmann-type classifier applicability and efficiency in uncovering variations and heterogeneity of germination response of Cistaceae species. Such an approach might offer synthetic answers on the pyrophytic vs. opportunistic debate on these obligate seeders. Data used are parts of broader experiments and measures, but they all focus on comparing collected, prepared, heat-treated, and germination and viability monitored seeds after a standard protocol from various individuals-populations-locations across the Mediterranean Basin. The protocol consisted of using mixtures of seeds collected from different individuals/species, several replicates/species, the same number of seeds/sample, comparison of germination and viability rates after a heat-treatment of $100{ }^{\circ} \mathrm{C}$ for $10 \mathrm{mins}$ in an air-forced oven; literature repeatedly reports this treatment as optimal or sub-optimal for the seeder species germination response (e.g., Moreno et al. 2011). Seeds of the control 
treatment with the same origin and replication procedures were not-heat-treated. Heat-treated and control samples were placed over two filter papers in $5.5 \mathrm{~cm}$ Petri dishes, moistened with $1.2 \mathrm{ml}$ of distilled water, and stored under specific controlled germination conditions per experiment (e.g., Luna et al. 2019; Luna 2020). In Troumbis' unpublished results, samples were randomly allocated in a walk-in growth chamber under glasshouse conditions. All samples were regularly checked for six to eight weeks for moisture saturation with deionized water (0 MPa water potential) (e.g., Chamorro and Moreno 2019), and those seeds/sample germinated were removed every two or three days until germination rate tailed off, and germination was not observed for consecutive days, depending on each experiment. Nongerminated seeds were controlled for viability following Moore's tetrazolium test (1985). The parameters $g_{i}, f_{i}, m_{i}, d_{i}$, and $v_{i}$, as well as the metrics $\theta$ and $c$, were measured and calculated to feed estimations of probability $P(+\mid+)$, i.e., the sensitivity or true positive germination rate, and $P(-\mid+)$, i.e., the specificity or false positive germination rate. To adapt these probability values to the classic ROC classification analysis procedure in IBM SPSS Statistics v26., I calculated the (1-specificity) value.

\subsection{Intra-population comparison: Cistus salviifolius in the Peloponnese}

I applied the standard heat-treatment and viability protocols presented in the previous section to estimate the various parameters of germination and physiology status of seeds of Cistus salviifolius, a species questioned as to its obligate seeding pyrophyte character, in several early publications (e.g., Trabaud 1995; Moretti et al. 2006; Troia and Laguna 2015). One hundred replicates of 25 mature seeds each, collected in late summer from different individuals, capsules, and neighboring populations (location-centered coordinates: $39^{\circ}$ N/2633’ E, North Peloponnese), were prepared (Troumbis and Trabaud 1987).

Values of the parameters $g_{i}, f_{i}, m_{i}, d_{i}$, and $v_{i}$ fed the Receiver Operating Characteristic (ROC) curve classification procedure in IBM SPSS Statistics v26. Cistus salviifolius pyrophytism hypothesis is valid in case of a significant difference from the null hypothesis $c$-metric $=0.5$ (Area Under the Curve or AUC in SPSS symbolism). Figure 3 presents the ROC curve results and the AUC significance level.

Results show that the heat-treatment effect on intra-population or inter-individual variation of the germination sensitivity-specificity of Cistus salviifolius at a location-scale give an excellent test (value $0.9<\mathrm{AUC} \leq 1$ ), that corresponds to acceptance of the pyrophytic character of the species (Figure 3B, C). On the contrary, the heat-treatment effect on viability is a worthless test $(\mathrm{AUC}<0.5)$. It is noticeable that $d_{i}$ and $v_{i}$ curves are inversely symmetric in the sensitivity - (1-specificity) plane. 


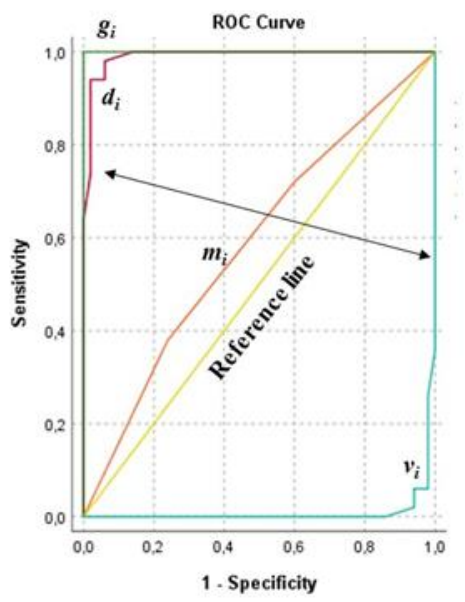

A: ROC curves for germination parameters

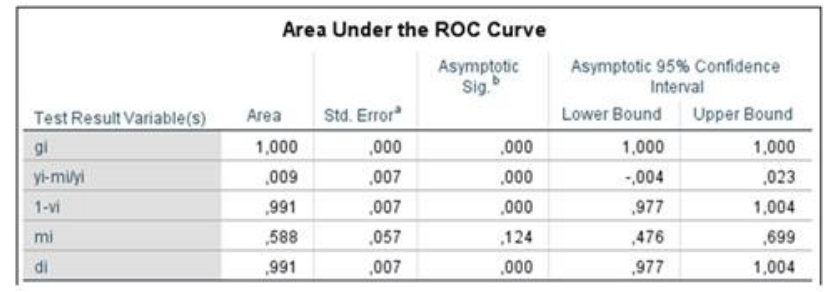

B: Summary of statistics for the ROC analysis procedure b: significance level for variables (null hypothesis $=0.5$ )

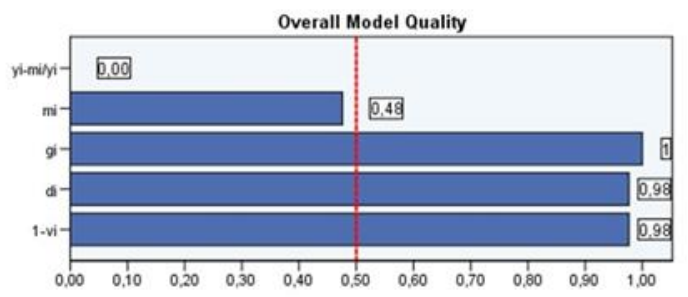
C: A good model has a value above 0.5
A value less than 0.5 indicates the model is no better than random
prediction

Figure 3: Receiver Operating Characteristic curves [A], the corresponding levels of significance for the single experiment model parameters $g_{i}, d_{i}, m_{i}$, and $v_{i}$ and $\left(1-v_{i}\right)[\mathrm{B}]$, and the overall model quality [C] as extracted from the IBM SPSS Statistics v26 dashboard. See text for more details.

\subsection{Intra-species comparisons: the case of Cistus salviifolius in the Mediterranean Basin}

In this exercise, I compared the five germination parameters and the basic model's two metrics obtained after the standard protocol for Cistus salviifolius from 13 locations across the Mediterranean Basin. Table 2 summarizes the environmental data regarding sites of seed provenance and references of published material. A data set on germination parameters is necessary for calculating comparable $\theta$ 's and c's or AUCs to run the meta-analysis procedure. To my best knowledge, although the question of seeds viability before- or after- treatment(s) is repeatedly discussed in the literature (e.g., Chamorro et al. 2017), combined data on germination and viability rates are rarely reported. An exemplary report to exploit in the present exercise is Luna (2020) supplementary material data set, which does report germination and viability percentages of 12 Cistaceae species seeds submitted to combinations of treatments. We further used data, published or graciously shared by authors from Luna et al. (2007); Luna and Chamorro (2016); Chamorro et al. (2017); Luna et al. (2019).

This exercise focuses on the variability in the germination response of Cistus salviifolius to general environmental conditions of the 13 sites of seed provenance, given that heat-shock does induce a significant increase in germination rates. Figure 4 presents $\Delta c$ metrics, i.e., differences in the c-metric or AUC values in ROC classification analysis between heat-treated and control seeds. Although site-specific data result from the interactive effects of multiple factors, the use of $\theta$ and c metrics allows for certain generalizations: (1a) viability of seeds decreases along the longitudinal gradient, as a general trend; and, (1b) heat-shock negatively affects the viability of seeds (see data from Chamorro et al. 2017). (2) Total annual precipitation positively influences both germination and viability rates. (3) The range of 
altitudinal variation does not uncover some discernable trend regarding both studied rates. (4) The mean annual temperature significantly affects the viability of seeds, especially after a heat-shock. salviifolius seeds were collected according to the standard protocol.

\begin{tabular}{|l|c|c|c|l|l|l|}
\hline Country & $\begin{array}{c}\text { Latitude } \\
(\mathbf{N})\end{array}$ & $\begin{array}{c}\text { Longitude } \\
(\mathbf{W})\end{array}$ & $\begin{array}{c}\text { Altitude } \\
(\mathbf{m})\end{array}$ & $\begin{array}{c}\text { Prec. } \\
\left(\mathbf{m m . y ^ { - 1 }}\right)\end{array}$ & $\begin{array}{c}\mathbf{T}(\boldsymbol{\mu} \\
\left.{ }^{\circ} \mathbf{C}\right)\end{array}$ & References \\
\hline Spain (C) & $39^{\circ} 48^{\prime}$ & $4^{\circ} 14^{\prime}$ & 550 & 600 & 12,5 & Luna 2020 \\
\hline Spain (C) & $39^{\circ} 45^{\prime}-39^{\circ} 54^{\prime}$ & $1^{\circ} 20^{\prime}-1^{\circ} 35^{\prime}$ & 25 & 600 & 12,5 & Luna et al. 2019 \\
\hline Spain (C) & $39^{\circ} 82^{\prime}$ & $-4^{\circ} 24^{\prime}$ & 530 & 480 & 14,7 & Chamorro et al. 2017 \\
\hline Spain (S) & $36^{\circ} 52^{\prime}$ & $-5^{\circ} 66^{\prime}$ & 400 & 650 & 17,6 & Chamorro et al. 2017 \\
\hline Spain (C) & $39^{\circ} 45^{\prime}-39^{\circ} 54^{\prime}$ & $1^{\circ} 20^{\prime}-1^{\circ} 35^{\prime}$ & 25 & 600 & 12,5 & Luna \& Chamorro 2016 \\
\hline France & $43^{\circ} 61^{\prime}$ & $3^{\circ} 40^{\prime}$ & 175 & 1300 & 11,9 & Chamorro et al. 2017 \\
\hline Italy & $40^{\circ} 33^{\prime}$ & $9^{\circ} 12^{\prime}$ & 350 & 965 & 13,2 & Chamorro et al. 2017 \\
\hline Greece 1 & $39^{\circ} 02^{\prime}$ & $26^{\circ} 61^{\prime}$ & 100 & 715 & 16,2 & Chamorro et al. 2017 \\
\hline Greece 2 & $38^{\circ} 55^{\prime}$ & $24^{\circ} 29^{\prime}$ & 250 & 430 & 17,2 & Troumbis (unpublished) \\
\hline Greece 3 & $38^{\circ} 10^{\prime}$ & $22^{\circ} 13^{\prime}$ & 200 & 475 & 18 & Troumbis (unpublished) \\
\hline Greece 4 & $39^{\circ}$ & $26^{\circ} 33^{\prime}$ & 35 & 645 & 17,8 & Troumbis (unpublished) \\
\hline Greece 5 & $35^{\circ} 17^{\prime}$ & $25^{\circ} 32^{\prime}$ & 180 & 475 & 19,9 & Troumbis (unpublished) \\
\hline Turkey & $36^{\circ} 60^{\prime}$ & $30^{\circ} 48^{\prime}$ & 70 & 700 & 12,3 & Chamorro et al. 2017 \\
\hline
\end{tabular}
270
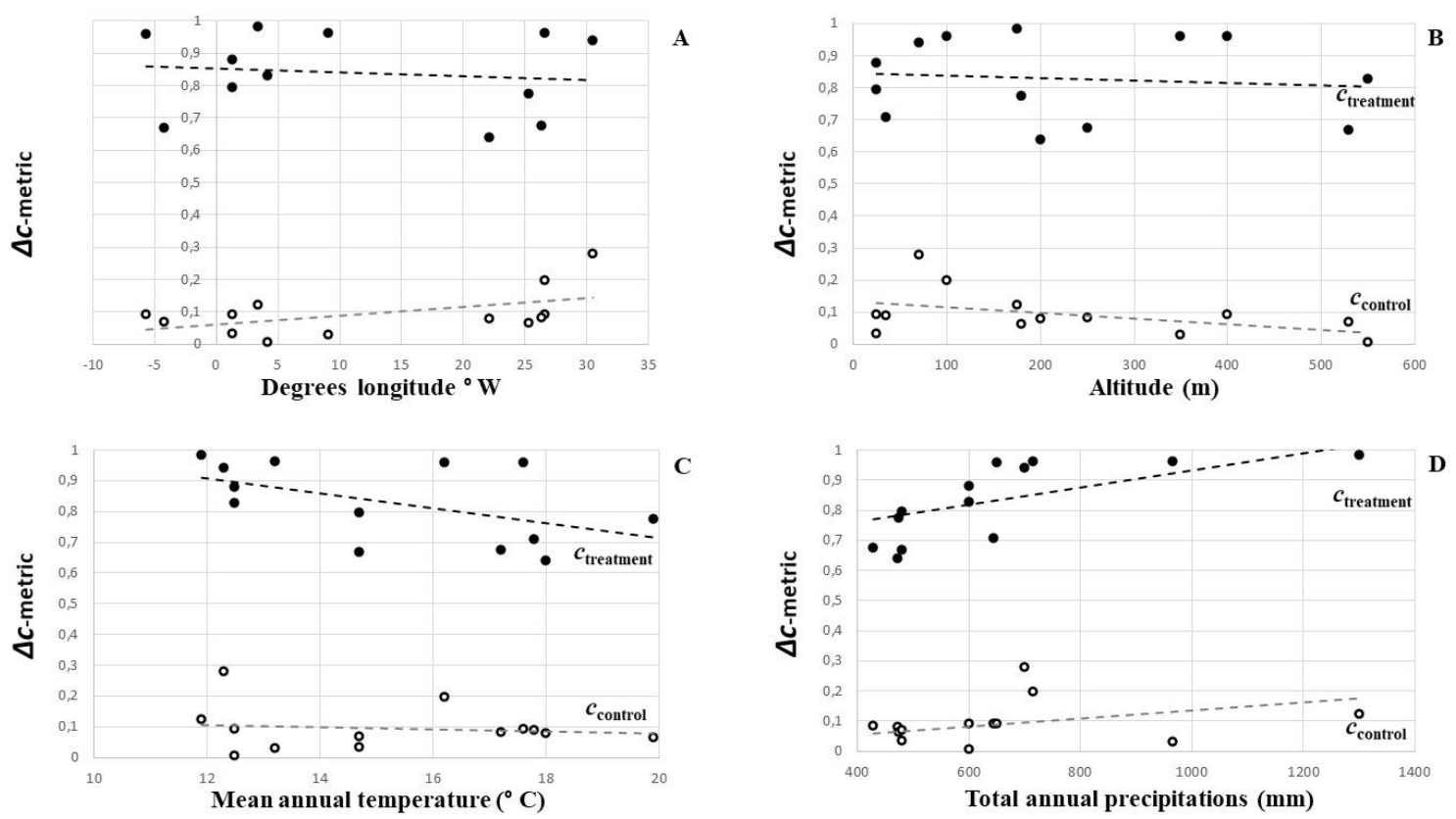

Figure 4: Effects of environmental factors upon the $\Delta \mathrm{c}$ metric (differences in the c-metric or AUC values in ROC classification analysis between heat-treated and control seeds) of Cistus salviifolius across the Mediterranean Basin. For details regarding site number, coordinates, altitude, and climatic data refer to Table 2 .

3.3.Intra-genus comparisons: the case of the genus Cistus

279 The genus Cistus is emblematic in Mediterranean Type shrublands typology (maquis and garrigues, e.g., Grove and Rackham (2001). It is also a common component of understorey 
vegetation in various Pinus-dominated forests, e.g., Trabaud (1983), especially under crowngap openings. The $\theta$ classifier and c-metric on data from three exemplary studies allow for uncovering that Cistus species could be ranked along a strong vs. light germination response to heat-shocks (e.g., data from Luna 2020). For instance, the goodness-of-fit to a linear regression of $\Delta c$ vs. species rank in the $R 1$ data set presents $R^{2}=0.96$, significant at $p<0.001$, and is compatible with the moving average $(\mathrm{lag}=2)$ trajectory with no-negative inflections in sequentially ranked species pairs comparisons.

This qualitative differentiation within the genus might be expressed quantitatively by introducing elasticity measures (Troumbis, under review). However, marked differences in $\Delta c$ 's between the 3 distinct data sets are observable in 5 over 8 studied Cistus species, i.e., $C$. clusii, C. laurifolius, C. ladaniferus, C. salviifolius, and C. populifolius (Figure 5).

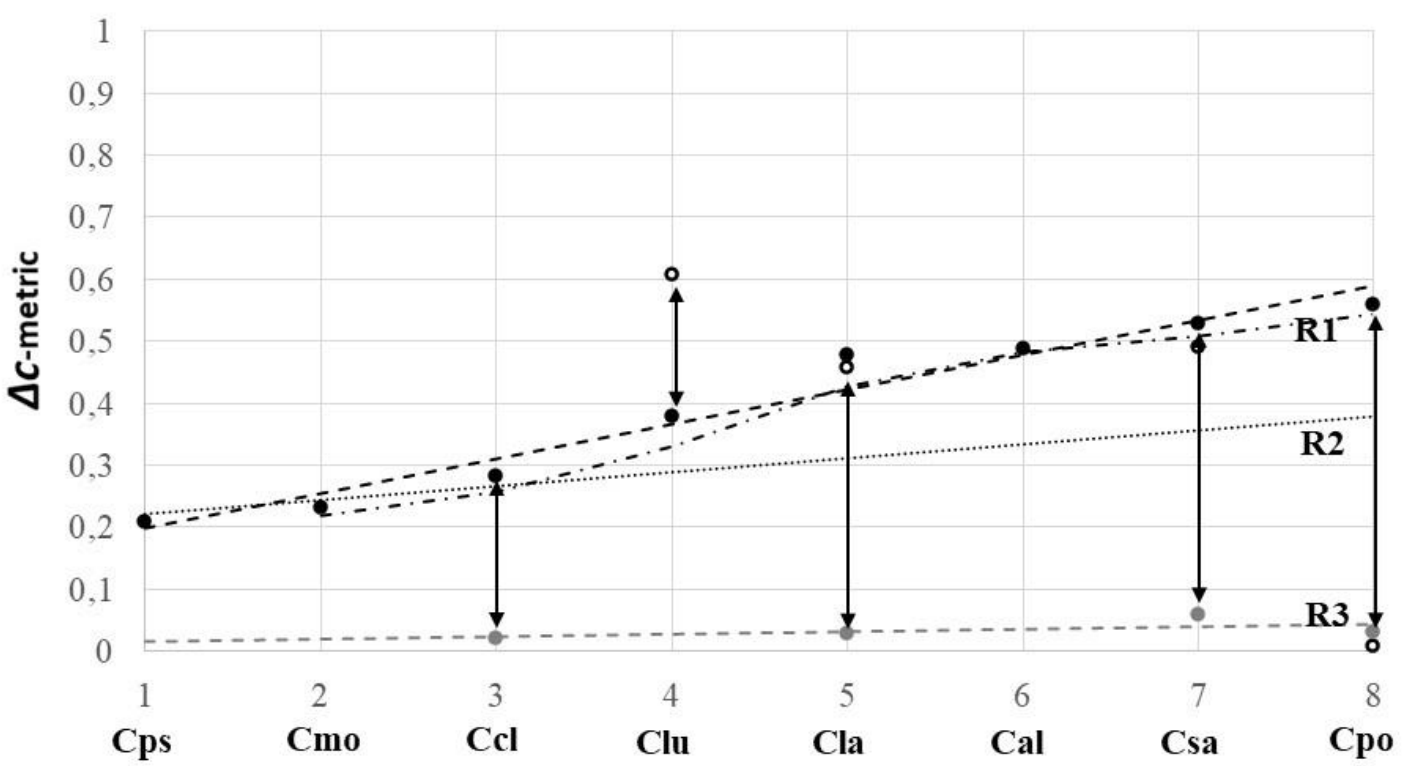

Genus Cistus

\subsection{Intra-family comparisons: the case of Cistaceae family}

Cistaceae family is present in the Mediterranean Basin with four more genera, besides Cistus, i.e., Fumana, Halimium, Helianthemum, and Tuberaria. The range of expansion, the variety of life forms, hybridization, local abundance, micro-habitat conditions, fire-proneness of their environment, and germination niche-breadth differ among Cistaceae (e.g., Luna and Moreno 2010). The data sets in the present meta-analysis comprise 8 Cistus species, 4 Halimium 
species, 2 Helianthemum species, 2 Fumana species, and 1 Tuberaria species. Figure 6 presents the Cistaceae $\theta$ and $\Delta \mathrm{c}$-metric classification results, species being sequentially ranked after the gradient of increasing $\Delta \mathrm{c}$ scale. The same $\Delta \mathrm{c} / \mathrm{rank}$ trend and similar variation between the 3 data sets are observed at the family level.

It is noticeable that except Halimium viscosum occupying the first rank position, species are ranked sequentially according to a genus suite, i.e., Cistus, Halimium, Fumana, Helianthemum, and Tuberaria. Further, Cistus sp. are shrubs or tall subshrubs, Halimium sp. are subshrubs, Helianthemum sp. and Fumana sp. are small subshrubs, and Tubraria sp. are perennial herbs. One can legitimately hypothesize that besides classic life-form and cycle effects, a body-size effect in the sense of physical distance between soil surface and seedcarrying capsules might materialize a particular aspect of fire pressure upon seed germination physiology.

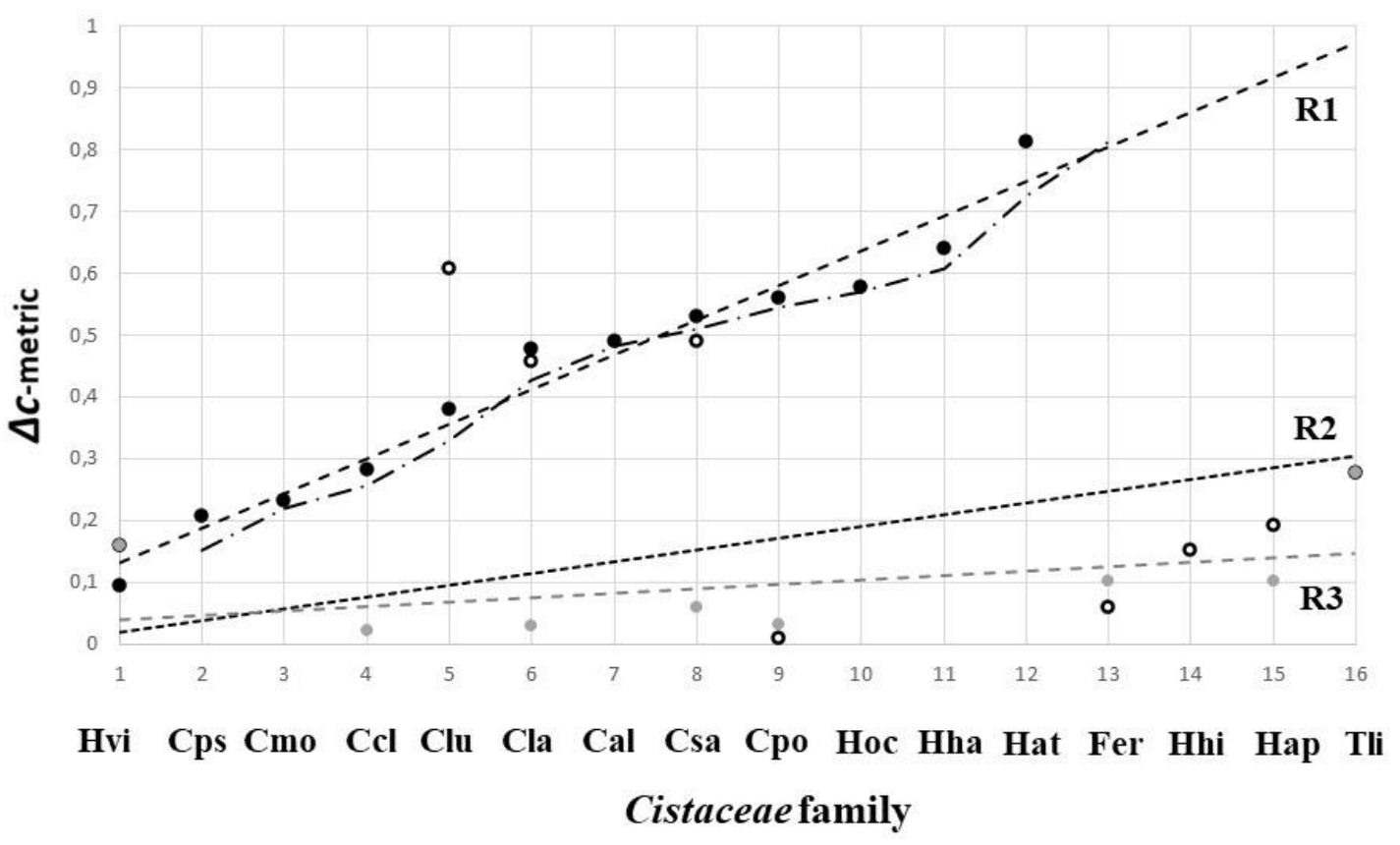

Figure 6: Ranking of Cistaceae species along a gradient of post-fire (heat-treatment) germination of seeds, using the $\theta$ classifier and the absolute value of c-metric. Species code: Hvi: Halimium viscosum; Cps: Cistus psilosephalus; Cmo: Cistus monspeliensis; Ccl: Cistus clusii; Clu: Cistus laurifolius; Cla: Cistus ladaniferus; Cal: Cistus albidus; Csa: Cistus salviifoius; Cpo: Cistus populifolius; Hoc: Halimium ocymoides; Hha: Halimium halimifolium; Hat: Halimium atriplicifolium; Fer: Fumana ericoides; Hhi: Helianthemum hirtum; Hap: Helianthemum apenninum; Tli: Tuberaria lignose. Solid black dashed line: linear regression line, slope $=0.06, \mathrm{R}^{2}=0.96, \mathrm{p}, 0.001$; dashed/dot line: moving average (lag $=$ 2). Data sources: R1: Luna (2020), available at https://doi.org/10.1038/s41598-020-62909-9. R2: Luna and Chamorro (2016), available https://doi.org/10.1017/S096025851600009X. R3: Luna et al. (2019), available at https://doi.org/10.1080/17550874.2019.1610916. 


\section{Discussion}

The discussion develops into two axes, technical and ecological. The first axis concerns the technical aspects of the meta-analysis framework I proposed. The second refers to the question of pyrophytism.

Meta-analysis using classification techniques like the Lehmann-type classifier developed here above and/or classic Receiver Operating Characteristic analysis is technically feasible. Its application limitations relate to data availability on seed populations' segments (e.g., dormant, viable, germinated et cetera) measured in various experiments or reported in the literature. For full use of its potential, disparate older -and eventually, new- datasets should be re-organized to feed data/seed bank segment for germination relative frequencies $\mathrm{P}(+\mid+), \mathrm{P}(-\mid+)$, and $\mathrm{P}(-\mid-)$ made available. In such an eventuality, ecological dichotomic questions, i.e., pyrophytism vs. opportunism, can transgress the limits of narrative evaluations and assessments.

This meta-analysis technique is flexible. For instance, I based comparisons along a taxonomic scale, from intra-population to intra-family variations, on $\theta$ and c-metric of the Lehmann-type model form $g=v^{\theta}$. As demonstrated in Section 2.2., the basic model can be extended and adapted to log- and logit- space, complexification by insertion of additional parameters or executed as a particular case of a different model family.

Our results indicate the kind of interpretation such a meta-analysis can support in the case of pyrophytism. Although heat-shock exerted upon hard-coated, dormant, and long-living seeds of the emblematic Cistaceae obligate seeders does break seed dormancy and initiate increased germination such a result is a case-specific population-level phenomenon that propagates along hierarchies of ecological organization levels and across taxonomic trees. Integration of empirical evidence from various levels of ecological and taxonomic differentiation tends instead supporting the interpretation that post-fire germination response of species does not lead to binary classification of Cistaceae as pyrophytes vs. opportunists or generalists. Instead, a perception of species arranged along a quantifiable gradient of reaction to heattriggered germination is closer to reality. One can accept a light-to-strong pyrophytism that integrates intra-species (Cistus salviifolius), intra-genus (Cistus), and intra-family (Cistus vs. Halimium, Cistaceae) heterogeneity. Further, the issue of an adverse effect of heat-shock upon seeds and seed banks of species considered as obligate seeding pyrophytes cannot be excluded if average viability is taken into account (refer to Cistus salviifolius examples in most Mediterranean locations, in Table S4 (Chamorro et al. 2017). This finding might just be a statistical artifact generated by the protocol procedure, but the question is worth assessing.

Situating this meta-analysis model and procedure within the climate change research, one should cautiously accept that obligate seeders' germination responses to fire are speciesidiosyncratic, involving biologically complex mechanisms (e.g., Siles et al. 2017) and significantly related to local environmental conditions (e.g., Chamorro et al. 2013). From the conservation, restoration, and fire management standpoints, as Céspedes et al. (2014) emphasized, burning season does not alter post-fire regeneration but could drive changes in species' rank/abundance distribution. Therefore, a conceptual departure from the original pyrophytic consideation, in the sense of C. Loehle (1987), towards a view that germination variability interacting with impacts of climate variability and change is necessary to promote more efficient ecosystem management and conservation planning in fire-prone ecosystems.

\section{Acknowledgments}


The author warmly thanks Professor Belén Luna's contribution for her comments and willingness to share data from previous experiments that critically improved this paper. Professors Panagiotis Dimitrakopoulos, N. Fyllas, and G. Kokkoris provided insightful comments on this paper's earlier version.

\section{References}

Camac J.S., Williams, R.J., Wahren C.H., Jarrad F., Hoffmann A.A.,Vesk P.A. (2015). Modeling rates of life form cover change in burned and unburned alpine heathland subject to experimental warming. Oecologia, 178 (2), 615-628. DOI: 10.1007/s00442-015-3261-2

Céspedes B., Torres I., Pérez B., Luna B., Moreno J.M. (2014). Burning season does not affect post-fire regeneration but fire alters the balance of the dominant species in a seederdominated Mediterranean shrubland. Applied Vegetation Science, 17, 711-725.

Chamorro D., Moreno J.M. (2019). Effects of water stress and smoke on germination of Mediterranean shrubs with hard or soft coat seeds. Plant Ecology, 220 ( 4-5), 511-521.

Chamorro D., Luna B., Moreno J.M. (2013). Germination response to various temperature regimes of four Mediterranean seeder shrubs across a range of altitudes. Plant Ecology, 214 (12), 1431-1441.

Chamorro D., Luna B., Moreno J.M. (2017). Germination responses to current and future temperatures of four seeder shrubs across a latitudinal gradient in western Iberia. American Journal of Botany, 104 (1), 83-91.

Chamorro D., Luna B., Ourcival, J.M., Kavgaci, A., Sirca C., Mouillot F., Arianoutsou M., Moreno J.M. (2017). Germination sensitivity to water stress in four shrubby species across the Mediterranean Basin. Plant Biology, 19, 23-31.

D'Antonio C., Flory S.L. (2017). Long-term dynamics and impacts of plant invasions. Journal of Ecology, 105 (6), SI 1459-1461.

Dijkstra, F.A., Adams, M.A. (2015). Fire Eases Imbalances of Nitrogen and Phosphorus in Woody Plants. Ecosystems, 18 (5), 769-779.

Driscoll D.A., Lindenmayer D.B., Bennett A.F., Bode M., Bradstock R.A., Cary G.J., Clarke M.F., Dexter N., Fensham R., Friend G., Gill M., James S., Kay G., Keith D.A., MacGregor C., Russell-Smith J., Salt D., Watson J.E.M., Williams R.J., York A. (2010). Fire management for biodiversity conservation: Key research questions and our capacity to answer them. Biological Conservation, 143 (9), 1928-1939. DOI: 10.1016/j.biocon.2010.05.026

Facelli J.M., Williams R., Fricker S., Ladd B. (1999). Establishment and growth of seedlings of Eucalyptus obliqua: Interactive effects of litter, water, and pathogens. Australian Journal of Ecology, 24 (5), 484-494 DOI: 10.1046/j.1440-169x.1999.00988.x

Fawcett T. (2006). An introduction to ROC analysis. Pattern Recognition Letters, 27 (8), 861-874.

Fyllas N.M.,Troumbis A.Y. (2009). Simulating vegetation shifts in north-eastern Mediterranean mountain forests under climatic change scenarios. Global Ecology and Biogeography, 18 (1), 64-77. 
Fyllas N.M., Dimitrakopoulos P.G., Troumbis A.Y. (2008). Regeneration dynamics of a mixed Mediterranean pine forest in the absence of fire. Forest Ecology and Management, 256 (8), 1552-1559.

Gagnon P.R., Passmore H.A., Platt W.J., Myers J.A., Paine C.E.T., Harms K.E. (2010). Does pyrogenicity protect burning plants? Ecology, 91 (12), 3481-3486.

Grove A.T., Rackham O. (2001). The nature of Mediterranean Europe. An ecological history. Yale University Press, New Haven, CT.

Hilborn R., Mangel M. (1997). The Ecological Detective - Confronting Models with Data. Princeton University Press, 315 p.

Holling H., Boehning W., Boehning D. (2012). Meta-analysis of diagnostic studies based upon SROC-curves: a mixed model approach using the Lehmann family. Statistical Modelling, 12 (4), 347-375.

Hudson A.R., Ayre D.J., Ooi M.K.J. (2015). Physical dormancy in a changing climate. Seed Science Research, 25 (2), SI 66-81.

Karavani A., Boer M.M., Baudena M., Colinas C., Diaz-Sierra R., Peman J., de Luis M., Enriquez-de-Salamanca A., de Dios V.R. (2018). Fire-induced deforestation in drought-prone Mediterranean forests: drivers and unknowns from leaves to communities. Ecological Monographs, 88 (2), 141-169. DOI: 10.1002/ecm.1285

Kuhnholtz-Lordat G. (1938). La terre incendiée. Essai d'agronomie compare; La Maison Carrée, Nimes, France, p. 36. (Burnt down land, deposited at https://agris.fao.org/agrissearch/search.do?recordID=US201300184078)

Le C.T. (2006). A solution for the most basic optimization problem associated with an ROC curve. Statistical Methods in Medical Research, 15, 571-584.

Lehmann E.L. (1990). Model specification: the views of Fischer and Neyman, and later developments. Statistical Science, 5, 2, 160-168.

Loehle C. (1987). Hypothesis testing in ecology: psychological aspects and the importance of theory maturation. - Quarterly Review of Biology, 62, 397-409.

Luna B. (2020). Fire and summer temperatures work together breaking physical seed dormancy. Scientific Reports, 10 (1), Article Number: 6031

Luna, B., Moreno J.M. (2010). Range-size, local abundance and germination niche-breadth in Mediterranean plants of two life-forms. Plant Ecology, 210 (1), 85-95.

Luna B., Chamorro D. (2016). Germination sensitivity to water stress of eight Cistaceae species from the Western Mediterranean. Seed Science Research, 26, 101-110.

Luna B., Chamorro D., Perez B. (2019). Effect of heat on seed germination and viability in species of Cistaceae. Plant Ecology and Diversity, 12 (2), 151-158.

Luna B., Moreno J.M., Cruz A., Fernandez-Gonzalez F. (2007). Heat-shock and seed germination of a group of Mediterranean plant species growing in a burned area: An approach based on plant functional types. Environmental and Experimental Botany, 60 (3), 324-333 DOI: $10.1016 /$ j.envexpbot.2006.12.014

Marlon J.R. (2020). What the past can say about the present and future of fire. Quaternary Research, 96, 66-87 Article Number: PII S0033589420000484 
Moore R.P. (1985). Handbook on tetrazolium testing; International Seed Testing Association, Zurich, Switzerland, 1985.

Moreno J.M., Zuazua E., Pérez B., Luna B., Velasco A., de Dios V.R. (2011). Rainfall patterns after fire differentially affect the recruitment of three Mediterranean shrubs. Biogeosciences, 8, 3721-3732.

Moretti M., Conedera M., Moresi R., Guisan A. (2006). Modelling the influence of change in fire regime on the local distribution of a Mediterranean pyrophytic plant species (Cistus salviifolius) at its northern range limit. Journal of Biogeography, 33 (8), 1492-1502. DOI: 10.1111/j.1365-2699.2006.01535.x

Ooi M.K.J. (2012). Seed bank persistence and climate change. Seed Science Research, 22 (S1), S53-S60

Rutter C.M., Gatsonis C.A. (2001). A hierarchical regression approach to meta-analysis of diagnostic test accuracy evaluations. Statistics in Medicine, 20 (19), 2865-2884.

Siles L., Müller M., Jana Cela J., Hernández H., Alegre L., Munné-Bosch S. (2017). Marked differences in seed dormancy in two populations of the Mediterranean shrub, Cistus albidus L. Plant Ecology \& Diversity, $10 \quad$ (2-3), 231-240. https://doi.org/10.1080/17550874.2017.1350765

Trabaud L. (1983). Post-fire development of the structure of some Mediterranean phytocenoses in Southern France. Annales des Sciences Forestieres, 40 (2), 177-196.

Trabaud L. (1994). Post-fire plant community dynamics in the Mediterranean Basin. In The role of fire in Mediterranean-type ecosystems Plant Ecology and Diversity; Moreno, J.M., Oechel W.C., Eds.; Springer-Verlag, New York; Ecological Studies 107, pp. 1-15.

Trabaud L. (1995). Germination of Mediterranean Cistus spp and Pinus spp and their reoccupation of disturbed sites. Revue Ecologie-Terre Vie, 50, 3-14.

Troia A., Laguna E. (2015). On the pyrophytism in the Mediterranean area. Journal of Arid Environments, 120, 1-3.

Troumbis A.Y, Trabaud L. (1986). Comparison of reproductive biological attributes of 2 Cistus species. Acta Oecologica-Oecologia Plantarum, 7 (3), 235-250.

Troumbis A.Y., Trabaud L. (1987). Seed bank dynamics of 2 Cistus species in Greek Mediterranean-Type Ecosystems. Acta Oecologica-Oecologia Plantarum, 8 (2), 167-179.

Troumbis A.Y., Trabaud L. (1989). Some questions about flammability in fire ecology. Acta Oecologica-Oecologia Plantarum, 10 (2), 167-175.

Troumbis A.Y., Memtsas D. (2000). Observational evidence that diversity may increase productivity in Mediterranean shrublands. Oecologia, 125 (1), 101-108.

Zavala M.A., Espelta J.M., Retana J. (2000). Constraints and trade-offs in Mediterranean plant communities: The case of holm oak-Aleppo pine forests. Botanical Review, 66 (1), 119-149

Zogas A., Kosman E., Sternberg M. (2020). Germination strategies under climate change scenarios along an aridity gradient. Journal of Plant Ecology, 13 (4), 470-477. 